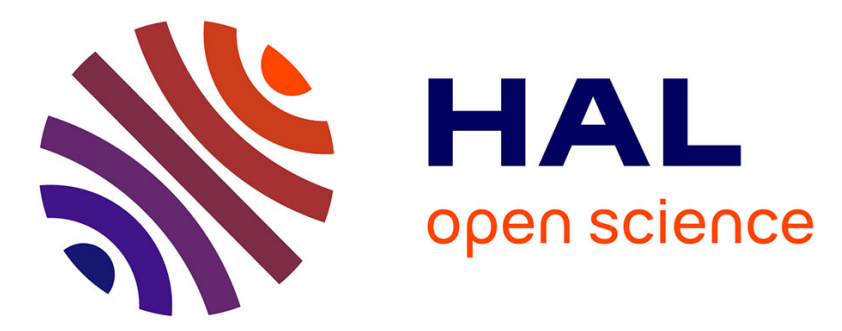

\title{
An adequate compositional encoding of bigraph structure in linear logic with subexponentials \\ Kaustuv Chaudhuri, Giselle Reis
}

\section{To cite this version:}

Kaustuv Chaudhuri, Giselle Reis. An adequate compositional encoding of bigraph structure in linear logic with subexponentials. 20th International Conference on Logic for Programming, Artificial Intelligence and Reasoning (LPAR), Nov 2015, Suva, Fiji. pp.146-161, 10.1007/978-3-662-48899-7_11. hal-01208362

\section{HAL Id: hal-01208362 \\ https://hal.inria.fr/hal-01208362}

Submitted on 2 Oct 2015

HAL is a multi-disciplinary open access archive for the deposit and dissemination of scientific research documents, whether they are published or not. The documents may come from teaching and research institutions in France or abroad, or from public or private research centers.
L'archive ouverte pluridisciplinaire HAL, est destinée au dépôt et à la diffusion de documents scientifiques de niveau recherche, publiés ou non, émanant des établissements d'enseignement et de recherche français ou étrangers, des laboratoires publics ou privés. 


\title{
An adequate compositional encoding of bigraph structure in linear logic with subexponentials
}

\author{
Kaustuv Chaudhuri ${ }^{1}$ and Giselle Reis ${ }^{1}$ \\ Inria \& LIX/École Polytechnique, France \\ $\{$ kaustuv.chaudhuri, giselle.reis\}@inria.fr
}

\begin{abstract}
In linear logic, formulas can be split into two sets: classical (those that can be used as many times as necessary) or linear (those that are consumed and no longer available after being used). Subexponentials generalize this notion by allowing the formulas to be split into many sets, each of which can then be specified to be classical or linear. This flexibility increases its expressiveness: we already have adequate encodings of a number of other proof systems, and for computational models such as concurrent constraint programming, in linear logic with subexponentials (SEL). Bigraphs were proposed by Milner in 2001 as a model for ubiquitous computing, subsuming models of computation such as CCS and the $\pi$ calculus and capable of modeling connectivity and locality at the same time. In this work we present an encoding of the bigraph structure in SEL, thus giving an indication of the expressive power of this logic, and at the same time providing a framework for reasoning and operating on bigraphs. Our encoding is adequate and therefore the operations of composition and juxtaposition can be performed on the logical level. Moreover, all the proof-theoretical tools of SEL become available for querying and proving properties of bigraph structures.
\end{abstract}

\section{Introduction}

Linear logic is excellent at counting elements of state since it interprets linear hypotheses as resources that are consumed upon use, and every linear hypothesis must be used in a proof. However, it is not particularly good at reasoning about relationships between elements. For example, an office building can have several rooms, with some rooms having a number of cubicles, and some cubicles containing several persons and computers. A precise description of this building must not only inventory all the rooms, cubicles, persons, and computers, but also state which component occurs inside which other components. In ordinary linear logic we can attempt to express the inclusion relation as a separate predicate, say a binary predicate $\operatorname{in}(x, y)$ that expresses that the entity with index $x$ is contained in that with index $y$, but such predicates are nearly impossible to treat linearly as they may be consulted and composed repeatedly. Yet, treating them non-linearly is also problematic: if a person moves from one cubicle to another, for example, the old placement needs to be invalidated, but there is no way to "consume" a non-linear resource in linear logic. 
Recently, a family of logics called subexponential logics (SEL) has emerged as a way to capture such intensional relationships between resources [12]. The idea is fairly old [6]: the exponential connectives ! and ? can be split into several different flavors, and the linear logic proof system would, a priori, make each version of the connectives independent of every other. If we impose a pre-order on them though, we can get a limited form of promotion where $?^{u} A$ entails $?^{v} A$ assuming the $u$ version of the exponentials are smaller than the $v$ version. In this view, $?^{u}$ corresponds, roughly, to a placement in the zone $u$, while ! ${ }^{u}$ corresponds to checking that every resource is placed in a zone larger than $u$. Subexponential logics are naturally much more expressive than just ordinary linear logic; for instance, even the propositional additive-free fragment is undecidable [5]. Moreover, they can easily express a wide spectrum of other deductive systems at a very high level of representational adequacy $[14,4,13]$.

In this paper, we consider the use of SEL as a logical framework for specifying bigraphs, a model of computation proposed by Milner in 2001 [10]. Bigraphs are a very general model, subsuming standard process calculi such as CCS [9] and the $\pi$-calculus [8]. Our use of SEL for this purpose has two primary goals:

- It gives a good formal indication of the expressive power of $S E L$ as a logical framework. If SEL can be used for bigraphs, it can probably also be used for any location-aware formalism. A number of such formalisms exist in the literature, such as ambients [3], brane calculi [2], and membrane computing [15].

- It provides an adequate syntactic treatment for bigraph structure. ${ }^{1}$ By adequate, we mean that any structural operation, such as composition and juxtaposition, can be performed directly on the syntactical representation. Bigraphs certainly have a well developed categorical semantics, but formalizing that semantics can be rather heavyweight. We show straightforward encoding and decoding functions from bigraphs to SEL sequents. Such a representation of bigraphs benefits from all the proof-theoretic tools available for querying and proving properties.

In relation to the second goal, another syntactic treatment for bigraphs can be found in [1]. The authors encode a bigraph and its reaction rules in the Concurrent LF ( $C L F)$ type theory, where both the formation and the reactions of bigraphs are encoded as rewrite rules. The encoding of locations is done by using a (linear) predicate has_child, and thus presents the problems previously mentioned. In particular, their use of a multi-set of atoms for encoding a bigraph makes it impossible to query a static structure, or prove its correctness, without damaging the structure itself. The proof that a bigraph is valid in [1] indeed consists of rewriting it to the empty set. Analogously, a containment check cannot be made without consuming the atom(s) encoding the parent relations.

In principle, we could use the same approach with rewriting rules to check for the correctness of bigraphs in our encoding, but we have decided to take

\footnotetext{
${ }^{1}$ We posit that, given the way computer science is evolving, the lack of formal and
} mechanized reasoning capabilities for any formalisms can be fatal. 
advantage of the expressiveness of subexponentials by having a more concise and conservative approach. In this work, we will encode bigraphs using fewer predicates than in [1] and in such a way that asking questions about the static structure will not cause any modifications to it. Our bigraph encoding changes only on actual operations, such as juxtaposition and compositions.

Our specific approach is to start with a sequent calculus for first-order classical SEL (Section 2.1). The classical dialect of SEL is used simply to keep the proof systems simple; our results extend to the intuitionistic dialect without any complications. The static definition of a bigraph is encoded in SEL as follows. The place graph of the bigraph is given in terms of atomic formulas placed in certain subexponential zones, and the subexponential signature encodes the parent relationship. The link graph of the bigraph is given in terms of eigenvariable parameters that are shared by every place that has a connection to a hyperedge. Finally, the inner and outer faces of the bigraph are expressed as purely linear formulas that are placed in the default zone of the SEL. This encoding will be an injection, i.e., it will have a right-inverse that will be able to extract the original bigraph from its sequent encoding. Furthermore, the static structure of the bigraph can be queried by trying to derive certain formulas. For example, to check whether a given node occurs inside (perhaps with multiple levels of nesting) a given place is tantamount to deriving a corresponding !-formula.

On top of this static description of a single bigraph, we define bigraph composition by first moving to a focused proof system for SEL, called SELF (Section 2.2) [11]. Focusing is a general mechanism for treating certain combinations of inferences in a (well behaved) sequent calulus as atomic derived inference rules. Bigraph juxtaposition in our encoding corresponds to a multiplicative composition of the two sequents describing the two bigraphs, and the composition of the bigraphs is given as a small theory on these sequents. We then run focused proof search on this sequent, extended with the composition theory, until quiescence, i.e., until no more focused steps are possible. The single unfinished leaf of the proof will then be the representation of the composed bigraph. This gives us the necessary representational adequacy theorem (Section 3.1).

\section{Background}

\subsection{Subexponential Logic (SEL)}

Subexponential logic (SEL) is a variant of linear logic with a family of exponential connectives - called subexponentials (a term coined in [12]) - that are indexed and arranged in a pre-order $[6,11]$. To keep things simple, we will use the classical dialect of $S E L$ in this paper. The grammar of formulas $(A, B, \ldots)$ is as follows:

$$
\begin{aligned}
& A, B, \ldots::=a|A \otimes B| 1|A \oplus B| 0|\exists x . A| !^{u} A \\
& |\neg a| A \gamma B|\perp| A \& B|\mathrm{\top}| \forall x . A \mid ?^{u} A \\
& \text { Literal mUltiplicative additive Quantified subexponential }
\end{aligned}
$$

Each column in the grammar is a De Morgan dual pair, and we indicate the dual of $A$ by $(A)^{\perp}$. The identifiers $a, b, \ldots$ range over atomic formulas, which 


$$
\begin{aligned}
& \frac{\vdash^{\mathcal{U}}, u: \neg a, a}{\text { init }} \frac{\vdash \Gamma^{\mathcal{U}}, \Delta_{1}, A \quad \vdash \Gamma^{\mathcal{U}}, \Delta_{2}, B}{\vdash \Gamma^{\mathcal{U}}, \Delta_{1}, \Delta_{2}, A \otimes B} \otimes \frac{\vdash \Gamma, A_{i}}{\vdash \Gamma^{\mathcal{U}}, 1}{ }^{1} \frac{\vdash \Gamma, A_{1} \oplus A_{2}}{\oplus_{i}} \frac{\vdash \Gamma,[t / x] A}{\vdash \Gamma, \exists x . A} \exists \\
& \frac{\vdash \Gamma, A, B}{\vdash \Gamma, A \gamma B} \ngtr \quad \frac{\vdash \Gamma}{\vdash \Gamma, \perp} \perp \frac{\vdash \Gamma, A \quad \vdash \Gamma, B}{\vdash \Gamma, A \& B} \& \quad \frac{\vdash \Gamma, \top}{\vdash \Gamma} \frac{\vdash \Gamma, A}{\vdash \Gamma, \forall x . A} \forall \frac{\vdash \Gamma, u: A}{\vdash \Gamma, ?^{u} A} ? \\
& \frac{(u \leq \vec{v}) \vdash \vec{v}: \vec{A}, C}{\vdash \Gamma^{\mathcal{U}}, \vec{v}: \vec{A}, !^{u} C} ! \quad \frac{(u \notin \mathcal{U}) \vdash \Gamma, A}{\vdash \Gamma, u: A} \text { derelict } \quad \frac{(u \in \mathcal{U}) \vdash \Gamma, u: A, A}{\vdash \Gamma, u: A} \text { copy }
\end{aligned}
$$

Fig. 1: A cut-free, one-sided, and zoned sequent calculus formulation of SEL. In the $\oplus_{i}$ rules, $i \in\{1,2\}$. In the $\forall$ rule, $x$ is not free in $\Gamma$.

are formulas of the form $\mathrm{p}\left(t_{1}, \ldots, t_{n}\right)$ where $\mathrm{p}$ stands for a predicate symbol and $t_{1}, \ldots, t_{n}$ are first-order terms. Each term is either a variable $(x, y, \ldots)$ or an application of the form $\mathrm{f}\left(s_{1}, \ldots, s_{m}\right)$ where $\mathrm{f}$ stands for a function symbol and the $s_{1}, \ldots, s_{m}$ are terms. Variables, predicates, and function symbols are assumed to be drawn from disjoint infinite sets and arities are fixed. We follow the usual syntactic convention of dropping the parentheses when the arity of a predicate or function symbol is 0 . The subexponential connectives $!^{u}$ and $?^{u}$ are indexed by subexponential labels $(u, w, \ldots)$ that belong to a subexponential signature.

Definition 1. A subexponential signature $\Sigma$ is a structure $\langle\Lambda, \mathcal{U}, \leq\rangle$ where:

$-\Lambda$ is a countable set of labels;

$-\mathcal{U} \subseteq \Lambda \uplus\{\varepsilon\}$, called the unbounded labels;

$-\leq$ is a pre-order on $\Lambda \uplus\{\varepsilon\}-i . e$. , it is reflexive and transitive-and $\leq$ upwardly closed with respect to $\mathcal{U}$, i.e., for any $u, w \in \Lambda \uplus\{\varepsilon\}$, if $u \in \mathcal{U}$ and $u \leq w$, then $w \in \mathcal{U}$; and

$-\varepsilon$ is $\leq$-minimal, i.e., for every $u \in \Lambda \uplus\{\varepsilon\}, \varepsilon \leq u$.

We say that any label in $\Lambda \uplus\{\varepsilon\} \backslash \mathcal{U}$ is bounded.

Intuitively, unbounded labels indicate formulas which can be weakened or contracted, while bound labels indicate linear formulas. We will assume an ambient subexponential signature $\Sigma$ unless we need to disambiguate particular instances of $S E L$, in which case we will use $\Sigma$ in subscripts. For instance, the set $\mathcal{U}_{\Sigma}$ stands for the unbounded labels of $\Sigma$.

The true formulas of SEL are derived from a sequent calculus proof system. There are many variants of such calculi in the literature; here, to keep things simple, we will use a zoned formulation where the members of a sequent are zoned formulas of the form $u$ :A where $A$ is a formula and $u \in \Lambda \uplus\{\varepsilon\}$. When it is unambiguous, we will drop the default zone label $\varepsilon$, i.e., write $\varepsilon: A$ as just $A$. A context $(\Gamma, \Delta, \ldots)$ is a non-empty multiset of zoned formulas, and $\Gamma, \Delta$ and $\Gamma, u: A$ stand as usual for the multi-set union of $\Gamma$ with $\Delta$ and $\{u: A\}$, respectively.

The inference rules for $S E L$ sequents are displayed in Figure 1. Most of the rules are shared between SEL and ordinary linear logic and will not be elaborated upon here; we will therefore explain only the peculiarities of SEL. 
Definition 2 (Notation). For $\vec{v}=\left[v_{1}, \ldots, v_{n}\right]$ and $\vec{A}=\left[A_{1}, \ldots, A_{n}\right]$ :

$-\vec{v}: \vec{A}$ stands for the context $v_{1}: A_{1}, \ldots, v_{n}: A_{n}$;

$-u \leq \vec{v}$ means that $u \leq v_{1}, \ldots$, and $u \leq v_{n}$; and

$-\vec{v} \in \mathcal{U}$ stands for $v_{1} \in \mathcal{U}, \ldots$, and $v_{n} \in \mathcal{U}$.

We write $\Gamma^{\mathcal{U}}$ for a context of the form $\vec{u}: \vec{A}$ where $\vec{u} \in \mathcal{U}$.

The rule for !, sometimes called promotion, has a side condition that checks that the label of the principal formula is less than the labels of all the other zoned formulas in the context that survive into the premise. This rule cannot be used if there are non-zoned formulas in the context, nor if the labels of some of the bound zoned-formulas are strictly smaller or incomparable with the subexponential label of the principal formula.

Theorem 1. The following rules are admissible for SEL.

$$
\begin{array}{cl} 
& \left(\forall w: C \in \Gamma^{\mathcal{U}} \cup \Delta_{1} \cup\{u: A\}, v \leq w\right) \\
\frac{\vdash-\Gamma^{\mathcal{U}}, \Delta_{1}, u: A \vdash \Gamma^{\mathcal{U}}, \Delta_{2}, v:(A)^{\perp}}{\vdash \Gamma^{\mathcal{U}}, A} \text { init* }_{1}, \Delta_{2} & \text { cut }
\end{array}
$$

Proof (sketch). The init* rule can be shown to be derivable by a straightforward structural induction on $A$, where reflexivity of $\leq$ is used in the cases for the subexponentials. For cut-admissibility, the standard permutative cut-reduction algorithm works. Transitivity of $\leq$ is used to justify the commutative cut case for !, while upward closure of $\leq$ with respect to $\mathcal{U}$ is used to justify permuting cuts past copy.

Subexponential logic is considerably more expressive than ordinary linear logic. Even the propositional additive-free fragment can be undecidable with as few as three subexponentials [5]. By choosing the appropriate signature it is possible to encode many proof systems [14] and various models of concurrency in concurrent constraint programming [13] in a natural way. This expressivity is a result of the ability of the ! rule to "check" the emptiness of certain zones in the sequent. Note that this rule can only be applied if all (linear) formulas are in bigger zones, which means that smaller or unrelated zones must be empty. It is natural then to view the zones in a sequent as locations where a formula has been stored (by the ? rule).

\subsection{Focusing (SELF)}

In our intended use of SEL as a specification logic for bigraphs, we will sometimes need to reason about sequences of inference steps that happen atomically. These derived or synthetic inference steps will be informed by focusing, which is a general technique for determining a normal form for a sequent calculus that eliminates many unnecessary branching points. A focused proof consists of an alternation of two phases. The asynchronous phase uses ${ }^{2}$ the inference rules in

\footnotetext{
${ }^{2}$ As usual in the view of the sequent calculus as a proof search formalism, we read inference rules from conclusion to premises.
} 


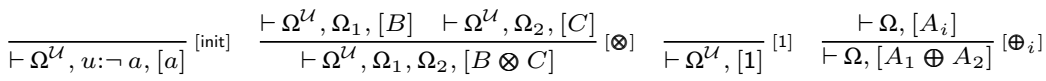

$$
\begin{aligned}
& \frac{\vdash \Omega,[[t / x] A]}{\vdash \Omega,[\exists x . A]}[\exists] \quad \frac{(u \leq \vec{w}) \quad \vdash \vec{w}: \vec{A}, C}{\vdash \Omega^{\mathcal{U}}, \vec{w}: \vec{A},\left[!^{u} C\right]}[!] \frac{\vdash \Omega, N}{\vdash \Omega,[N]} \text { [release] }
\end{aligned}
$$

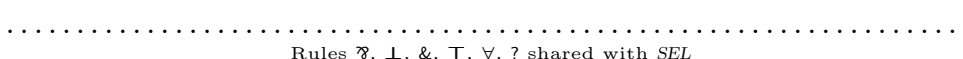

$$
\begin{aligned}
& \frac{(u \notin \mathcal{U}) \vdash \Omega,[A]}{\vdash \Omega, u: A} \text { Idecide } \quad \frac{(u \in \mathcal{U}) \vdash \Omega, u: A,[A]}{\vdash \Omega, u: A} \text { udecide }
\end{aligned}
$$

Fig. 2: The SELF inference system, a focused version of SEL. The context $\Omega$ is such that if $A \in \Omega$ then $A$ is a positive formula or a negated atom.

the second line of Figure 1 that are invertible and can therefore always be safely applied. When no such rule is applicable, the proof enters a synchronized phase by selecting a formula for focus; this formula is decomposed under focus using the rules in the first line of Figure 1 until no such rule applies, in which case the phase switches back to asynchronous again. To be a bit more explicit, we add a new kind of focused sequent of the form $\vdash \Gamma$; $[A]$ where $A$ is the focused formula.

The inference rules for the focused version of $S E L$, called $S E L F$, are given in Figure 2. These rules are based on a division of the formulas of SEL into positive formulas $(P, Q, \ldots)$ that come from the first line of (1), and negative formulas $(N, M, \ldots)$ that come from the second line. We use the convention that $\Omega$ stands for a context that consists of zoned formulas where if the zone label is $\varepsilon$ then the corresponding formula is positive or a literal. As before $\Omega^{\mathcal{U}}$ stands for a context of the form $\vec{u}: \vec{A}$ where $\vec{u} \in \mathcal{U}$. There are two decision rules, Idecide and udecide, for focusing on a zoned formula with a bound label and an unbound label respectively. The focus persists through to the immediate subformula in the synchronous phase rules $[\otimes],\left[\oplus_{i}\right]$, and $[\exists]$. Ultimately, the focused formula becomes negative or a !-formula, in which case focus is released and the asynchronous phase begins, where the inference rules are identical to those of SEL. Finally, when no more asynchronous rules are available, a decision rule is used to enter the focused phase again.

Theorem 2 (Soundness and Completeness of SELF). The sequent $\vdash \Gamma$ is derivable in SELF if and only if it is derivable in SEL.

Proof (sketch). Soundness is immediate, since if we interpret $\vdash \Omega,[A]$ as $\vdash \Omega, A$ then we get a $S E L$ proof from a $S E L F$ proof. For completeness, we can easily adapt any of the existing proofs for related systems, such as [11, chapter 5].

\section{Bigraphs in SEL}

Bigraphs were proposed by Milner [10] as a model for ubiquitous computing. It tries to fill in a gap between the current state of computational systems, which involves communications on a global scale and pervasive computing, and the available tools to model and reason about such systems. In bigraphs, locality and connectivity of agents are treated independently, and they might be equipped with reaction rules (i.e., rewriting) for reconfiguration. It has been shown that bigraphs 


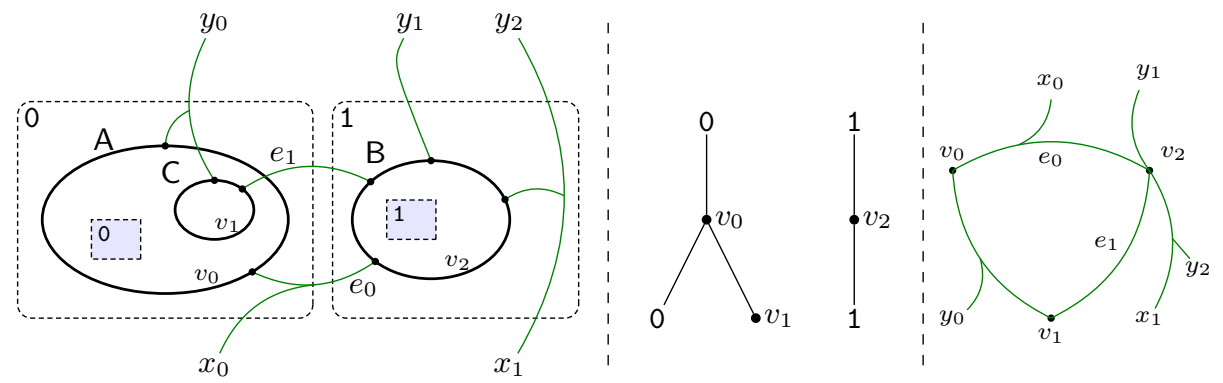

Fig. 3: A bigraph and its place and link graphs.

subsume the calculus of communicating systems (CCS) [9] and $\pi$-calculus [8]. Most definitions and examples in this section were taken from [9].

A bigraph is a combination of two graphs (hence the name): a place graph and a link graph. Both have in common the set of vertices, but the place graph is a set of trees while the link graph is a hypergraph (i.e., one edge can connect any number of nodes). Figure 3 is an example of a bigraph and its components, each representing orthogonally the concepts of locality and connectivity.

Each node takes the role of a specific control that determines its ports. The controls available for a bigraph are defined in its signature, which does not have to be finite or even denumerable.

Definition 3 (Bigraph signature). A signature $\mathcal{K}$ is a set of elements called controls, each with an arity $(\operatorname{ar}(K))$. The signature also determines which controls are atomic and which of the non-atomic controls are active.

Depending on the system being represented, such signatures can be augmented with, for example, sorts that enforce what kind of controls a non-atomic control can contain, or signs that make sure only ports of opposite polarities are connected. For a discussion on adding sorts to our encoding, see Section 4. Given a signature, we can formally define a bigraph.

Definition 4 (Bigraphs). $A$ bigraph $\mathcal{B}$ over the signature $\mathcal{K}$ is defined as:

$$
\mathcal{B}=(V, E, \text { ctrl, prnt }, \text { link }):\langle m, X\rangle \rightarrow\langle n, Y\rangle .
$$

- $V$ is a set of node names.

$-E$ is a set of edge names.

- $m$ is the number of sites (i.e., holes which can accommodate other bigraphs).

- $X$ is a set of inner names.

- $n$ is the number of roots (i.e., which can be accommodated in other bigraphs).

- $Y$ is a set of outer names.

- ctrl $: V \rightarrow \mathcal{K}$ defines the role of each node.

- prnt : $m \uplus V \rightarrow n \uplus V$ defines the parent relation, or the place graph.

- link : $X \uplus P \rightarrow Y \uplus E$ defines the connectivity, or the link graph, where $P=\{(v, i) \mid 1 \leq i \leq \operatorname{ar}(\operatorname{ctrl}(v))\}$, i.e, $(v, i)$ is the $i^{\text {th }}$ port of node $v$. 
The sets of names $V, E, X$, and $Y$ must be pairwise disjoint. The terms $m$ and $n$ are treated notationally as sets of all strictly smaller natural numbers, i.e., the sites are numbered $0,1, \ldots, m-1$, and likewise for the roots.

For the bigraph of Figure 3, we have $\mathcal{K}=\{\mathrm{A}: 2, \mathrm{~B}: 4, \mathrm{C}: 2\}$ and:

$$
\begin{aligned}
& V=\left\{v_{0}, v_{1}, v_{2}\right\} \quad E=\left\{e_{0}, e_{1}\right\} \quad X=\left\{x_{0}, x_{1}\right\} \quad Y=\left\{y_{0}, y_{1}, y_{2}\right\} \quad m=n=2 \\
& \text { ctrl prnt link } \\
& v_{0} \mapsto \mathrm{A} \quad 0 \mapsto v_{0} \quad x_{0} \mapsto e_{0} \quad\left(v_{1}, 2\right) \mapsto e_{1} \\
& v_{1} \mapsto \mathrm{C} \quad 1 \mapsto v_{2} \quad x_{1} \mapsto y_{2} \quad\left(v_{2}, 1\right) \mapsto y_{1} \\
& v_{2} \mapsto \mathrm{B} \quad v_{0} \mapsto 0 \quad\left(v_{0}, 1\right) \mapsto y_{0} \quad\left(v_{2}, 2\right) \mapsto y_{2} \\
& v_{1} \mapsto v_{0} \quad\left(v_{0}, 2\right) \mapsto e_{0} \quad\left(v_{2}, 3\right) \mapsto e_{0} \\
& v_{2} \mapsto 1 \quad\left(v_{1}, 1\right) \mapsto y_{0} \quad\left(v_{2}, 4\right) \mapsto e_{1}
\end{aligned}
$$

\subsection{Encoding bigraphs}

Given a bigraph $\mathcal{B}$, we will show in this section how to encode it using a SEL sequent. Intuitively, each place is represented by a subexponential index; indices are related according to prnt in the place graph. Next, each place which has an assigned control (i.e., the actual nodes) will be represented by a predicate, conveniently named control and placed in the zone corresponding to its parent. The links are encoded by using constants or variables that are shared among the controls if they are linked together. We will use a typewriter font to denote constants in our encoding.

Definition 5 (Encoding function). Let $\mathcal{B}=(V, E$, ctrl, prnt, link $):\langle m, X\rangle \rightarrow$ $\langle n, Y\rangle$ be a bigraph over a signature $\mathcal{K}$, then:

- Term and atomic formulas:

- id is a unique identifier.

- Each element of $V, E$, and $\mathcal{K}$ is a constant.

- Each element of $X$ and $Y$ is a variable.

- control $(\mathrm{K}, \mathrm{v}, L)$ is an atomic formula, where $L$ is a list of size ar $(\mathrm{K})$.

- in $(x, l, \mathrm{id})$ and out $(y, \mathrm{id})$ are atomic formulas, where $l$ is a constant or variable corresponding to an element $e \in E \cup Y$.

- site(id) and root(id) are atomic formulas.

- Subexponential signature $\Sigma=\langle\Lambda,\{\infty\}, \leq\rangle$ :

- Each node name is duplicated as a label, i.e., $V \subseteq \Lambda$.

- $r_{0}, \ldots, r_{n-1} \in \Lambda \backslash V$ are subexponential variables representing the roots.

- $\forall(a, b) \in$ prnt. $b \leq a \in \Sigma .^{3}$

The function $E(-)$ maps $\mathcal{B}$ to the $S E L_{\Sigma}$ sequent:

$$
\begin{aligned}
\vdash_{\Sigma} & \{\operatorname{in}(x, \operatorname{link}(x), \mathrm{id}) \mid x \in X\},\{\operatorname{out}(y, \mathrm{id}) \mid y \in Y\} \\
& \left\{r_{i}: \operatorname{root}(\mathrm{id}) \mid 0 \leq i \leq n-1\right\},\{\operatorname{prnt}(i): \operatorname{site}(\mathrm{id}) \mid 0 \leq i \leq m-1\}, \\
& \{\operatorname{prnt}(\mathrm{v}): \operatorname{control}(\operatorname{ctrl}(\mathrm{v}), \mathrm{v},[\operatorname{link}((\mathrm{v}, 1)), \ldots, \operatorname{link}((\mathrm{v}, \operatorname{ar}(\operatorname{ctrl}(\mathrm{v}))))]) \mid \mathrm{v} \in V\} .
\end{aligned}
$$

Note that all $x, y$ and $r_{i}$ are free in the resulting sequent.

\footnotetext{
${ }^{3}$ If $a$ or $b$ is a natural number $1 \leq i \leq m$ representing a root, than we map it to $r_{i}$ in the subexponential signature.
} 
As an example, if $\mathcal{B}$ is the bigraph of Figure 3, then $E(\mathcal{B})$ is the sequent:

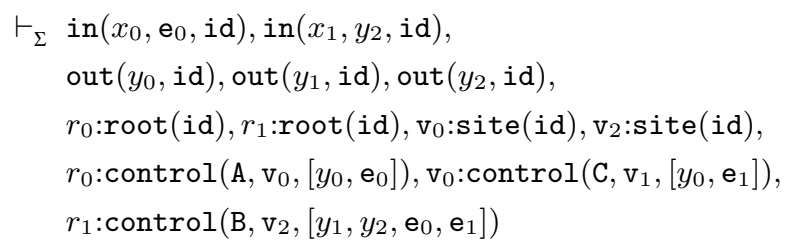

Where $\Sigma=\left\langle\left\{r_{0}, r_{1}, \mathrm{v}_{0}, \mathrm{v}_{1} \cdot \mathrm{v}_{2}, 0,1\right\},\{\infty\},\left\{r_{0} \leq \mathrm{v}_{0} \leq \mathrm{v}_{1} ; \mathrm{v}_{0} \leq 0 ; r_{1} \leq \mathrm{v}_{2} \leq 1\right\}\right\rangle$.

We will now show that it is possible to define a function that takes a sequent encoding a bigraph and returns the bigraph itself. In order to be a bigraph encoding, a SEL sequent must be of a specific shape and satisfy some conditions which are specified in the definition that follows.

Definition 6 (Decoding function). Let $S$ be the following SEL sequent:

$$
\begin{gathered}
\vdash_{\Sigma}\left\{\text { in }\left(x_{i}, l_{x_{i}}, \text { id }\right) \mid 0 \leq i \leq n_{x}-1\right\},\left\{\text { out }\left(y_{i}, \text { id }\right) \mid 0 \leq i \leq n_{y}-1\right\}, \\
\quad\left\{r_{i}: \operatorname{root}(\mathrm{id}) \mid 0 \leq i \leq n-1\right\},\left\{p_{i}: \operatorname{site}(\mathrm{id}) \mid 0 \leq i \leq n_{v}-1\right\}, \\
\quad\left\{p_{i}: \operatorname{control}\left(\mathrm{K}_{j}, \mathrm{v}_{i}, L_{j}\right) \mid 0 \leq i \leq n_{v}-1,0 \leq j \leq n_{k}-1\right\}
\end{gathered}
$$

for integers $n, n_{x}, n_{y}, n_{v}$ and $n_{k}$, satisfying the following conditions:

$-x_{i}, y_{i}$ and $r_{i}$ are free variables.

- $L_{i}$ is a list of variables and constants.

- $l_{x_{i}}$ is a variable that occurs as the first argument of an out predicate or a constant that occurs in a $L_{j}$ for some $j$.

- $p_{i}$ is either a variable that occurs as a label for some root, or a constant that occurs as the second argument for some control predicate different than $\mathrm{v}_{i}$.

- $p_{i} \leq v_{i} \in \Sigma$ for each $p_{i}$ labeling a control $\left(\mathrm{K}_{j}, \mathrm{v}_{i}, L_{j}\right)$.

- $p_{i} \leq i \in \Sigma$ for each $p_{i}$ labeling a site(id).

- the relation $\leq$ in $\Sigma$ defines a tree.

Then, $D(S)$ is a bigraph $(V, E$, ctrl, prnt, link $):\langle m, X\rangle \rightarrow\langle n, Y\rangle$ over a signature $\mathcal{K}$, where:

- $\mathcal{K}$ contains all the $\mathrm{K}_{j}$ with $\operatorname{ar}\left(\mathrm{K}_{j}\right)=\left|L_{j}\right|$;

$-V=\left\{v_{i} \mid 0 \leq i \leq n_{v}-1\right\}$;

- $E$ is the set of constants occurring in the lists $L_{i}$;

$-\operatorname{ctrl}\left(v_{i}\right)=\mathrm{K}_{j}$ if $l: \operatorname{control}\left(\mathrm{K}_{j}, \mathrm{v}_{i}, L\right) \in S$ (for any $\left.L\right)$;

- prnt is the same as the $\leq$ relation in $\Sigma \backslash\{\varepsilon, \infty\}$;

$-\operatorname{link}\left(x_{i}\right)=l_{x_{i}}$ if in $\left(x_{i}, l_{x_{i}}\right.$, id $)$;

$-\operatorname{link}((v, i))=l$ if control $(-, \mathrm{v}, L) \in S$ and $L[i]=l$;

$-X=\left\{x_{i} \mid 0 \leq i \leq n_{x}-1\right\}$;

$-Y=\left\{y_{i} \mid 0 \leq i \leq n_{y}-1\right\} ;$ and

$-m$ is the number of site(id) predicates.

We will now show that $D$ is a left-inverse of $E$. 
Theorem 3 (Representational adequacy). Let $\mathcal{B}$ be a bigraph, $S=E(\mathcal{B})$ and $\mathcal{B}^{\prime}=D(S)$. Then $\mathcal{B}=\mathcal{B}^{\prime 4}$.

Proof (sketch). For each element $V, E$, prnt, ctrl, link, $m, n, X$ and $Y$ of a bigraph, $E$ maps it to a certain element of $S$ and $D$ maps it back.

Theorem 4. The function $D$ is surjective, i.e., for every bigraph $\mathcal{B}$, there exists a SEL sequent $S$ such that $D(S)=\mathcal{B}$.

Proof. By the totality of $E$, we know that for every bigraph $\mathcal{B}$, there exists a sequent $S=E(\mathcal{B})$. From Theorem 3 , we know that $D(S)=\mathcal{B}$.

An encoding of a bigraph in SEL will allow us to use the tools available in the logic to reason about it. In particular, we can use the informations about subexponentials to easily deduce when a node $v_{i}$ is contained in $v_{j}$, even if $v_{j}$ is not $v_{i}$ 's immediate parent.

Theorem 5. Let $\mathcal{B}$ be a bigraph over a signature $\mathcal{K}$. Assume that $\Sigma$ is the subexponential signature generated by $E(\mathcal{B})$. If the sequent $\vdash_{\Sigma} !^{\mathrm{v}_{j}}(A)^{\perp} 8 ?^{\mathrm{v}_{i}} A$ is provable for an arbitrary $A$, then the node $v_{i}$ is contained in node $v_{j}$ (with an arbitrary number of nestings) in $\mathcal{B}$.

Proof. While deriving this sequent in SEL, the promotion rule generates the side condition $\mathrm{v}_{j} \leq \mathrm{v}_{i}$. Since $\leq$ is transitive, and the parent relation is the kernel of $\leq$, we have $\operatorname{prnt}^{k}\left(v_{i}\right)=v_{j}$ for some $k$, so $v_{i}$ is contained in $v_{j}$ in $\mathcal{B}$.

\subsection{Juxtaposition}

One of the two basic combination mechanisms for bigraphs is juxtaposition, which places two disjoint bigraphs "side-by-side". The formal definition can be found in [10, Definition 2.7]. At a basic level, this is easily achieved in terms of SEL sequents as we merely have to take the multiset join of the two encodings of the constituent bigraphs. However, it is not entirely trivial, since the roots and sites need to be renumbered.

Definition 7 (Juxtaposition). Given $\Sigma_{1}=\left\langle\Lambda_{1}, \mathcal{U}_{1}, \leq_{1}\right\rangle$ and $\Sigma_{2}=\left\langle\Lambda_{2}, \mathcal{U}_{2}, \leq_{2}\right\rangle$ with $\Lambda_{1} \cap \Lambda_{2}=\{\infty\}$ that are the subexponential signatures for the encoding of two bigraphs with interfaces $\left\langle n_{1}, X_{1}\right\rangle \rightarrow\left\langle m_{1}, Y_{1}\right\rangle$ and $\left\langle n_{2}, X_{2}\right\rangle \rightarrow\left\langle m_{2}, Y_{2}\right\rangle$ respectively, the signature $\Sigma_{1} \mid \Sigma_{2}$ with corresponding substitution on locations $\sigma$ is defined to be the structure $\langle\Lambda, \mathcal{U}, \leq\rangle$ where:

$-\sigma=\left\{r_{n_{1}+i} / r_{i} \mid 0 \leq i<n_{2}\right\} .{ }^{5}$

$-\Lambda=\Lambda_{1} \cup \Lambda_{2} \sigma$ and $\mathcal{U}=\mathcal{U}_{1} \cup \mathcal{U}_{2} \sigma$.

$-x \leq y$ iff $x \leq_{1}$ y or $x \sigma \leq_{2} y \sigma$.

(We use $\mid$ instead of $\otimes$ to indicate juxtaposition to avoid confusion with the SEL connective.)

\footnotetext{
${ }^{4}$ We always consider bigraphs to be equal up to the renaming of elements.

${ }^{5} t / s$ denotes the substitution of $s$ by $t$.
} 
Theorem 6 (Adequacy of juxtaposition). Given disjoint bigraphs $\mathcal{B}_{1}$ and $\mathcal{B}_{2}$, let $E\left(\mathcal{B}_{1}\right)$ be $\vdash_{\Sigma_{1}} \Gamma_{1}$ and $E\left(\mathcal{B}_{2}\right)$ be $\vdash_{\Sigma_{2}} \Gamma_{2}$, such that both encodings use the same identifier id. Let $\sigma$ be the substitution for $\Sigma_{1} \mid \Sigma_{2}$. Then: $E\left(\mathcal{B}_{1} \mid \mathcal{B}_{2}\right)=$ $\vdash_{\Sigma_{1} \mid \Sigma_{2}} \Gamma_{1},\left(\Gamma_{2} \sigma\right)$.

Proof. Immediate by inspection..

Note that we only need to renumber the roots, since the sites are implicitly numbered in our encoding. This is a consequence of linearity (to distinguish sites) and subexponentials (used to name the immediate parent of every site).

\subsection{Composition}

The interface of a bigraph, i.e., $\langle m, X\rangle \rightarrow\langle n, Y\rangle$, determines the available links and places for composition with another bigraph. A bigraph $\mathcal{B}_{1}:\left\langle m_{1}, X_{1}\right\rangle \rightarrow$ $\left\langle n_{1}, Y_{1}\right\rangle$ can be composed with a bigraph $\mathcal{B}_{2}:\left\langle m_{2}, X_{2}\right\rangle \rightarrow\left\langle n_{2}, Y_{2}\right\rangle$ if $n_{1}=m_{2}$ and $Y_{1}=X_{2}$. This means that $\mathcal{B}_{1}$ has $n_{1}$ roots that will be placed inside $\mathcal{B}_{2}$ 's $m_{2}$ sites, and all $\mathcal{B}_{1}$ 's outer names $Y_{1}$ will be connected with $\mathcal{B}_{2}$ 's inner names $X_{2}$. This composition is denoted by $\mathcal{B}_{2} \circ \mathcal{B}_{1}$. For a formal definition of bigraph composition, see [10, Definition 2.5]. Such compositions can be adequately captured in our framework by a focused derivation of formulas which will encode the operations of (1) connecting links and (2) placing roots inside sites.

Let $S_{1}=E\left(\mathcal{B}_{1}\right)$ be the sequent $\vdash_{\Sigma_{1}} \Gamma_{1}$ and $S_{2}=E\left(\mathcal{B}_{2}\right)$ be $\vdash_{\Sigma_{2}} \Gamma_{2}$ such that the names in $S_{1}$ do not clash with those of $S_{2} \cdot{ }^{6}$. The composition of these into one bigraph will require a common subexponential signature, defined below.

Definition 8. Let $\Sigma_{1}=\left\langle\Lambda_{1}, \mathcal{U}_{1}, \leq_{1}\right\rangle$ and $\Sigma_{2}=\left\langle\Lambda_{2}, \mathcal{U}_{2}, \leq_{2}\right\rangle$ be such that $\Lambda_{1} \cap$ $\Lambda_{2}=\{\infty\}$. Then, $\Sigma_{2} \circ \Sigma_{1}$ is the signature $\langle\Lambda, \mathcal{U}, \leq\rangle \sigma$ such that

$$
\Lambda=\Lambda_{1} \cup \Lambda_{2}, \quad \mathcal{U}=\mathcal{U}_{1} \cup \mathcal{U}_{2}, \quad \leq=\leq_{1} \cup \leq_{2}, \quad \sigma=\left\{\mathrm{v}_{i} / r_{j} \mid v_{i} \in \Lambda_{2} \text { and } r_{j} \in \Lambda_{1}\right\} .
$$

The substitution $\sigma$ defines a map between sites and roots. ${ }^{7}$ In the subexponential signature, this means that the root $r_{j}$ will be instantiated with the index $\mathrm{v}_{i}$ at the same time that one of its successors (i.e., a site) is removed, which is equivalent to replacing a numeric successor of $v_{i}$ by the subtree of $r_{j}$.

The composed subexponential signature yields the definition of the formulas encoding the operations of linking and placing. Consider the sequent:

$$
\vdash_{\Sigma} \Gamma_{1}, \Gamma_{2}, \infty: \exists e \cdot(\operatorname{in}(e, e, \text { id } 2))^{\perp} \otimes(\operatorname{out}(e, \text { id } 1))^{\perp} \otimes \perp
$$

where all free variables are interpreted as existential variables, i.e., they are unified with terms in applications of the [init] rule. We will henceforth denote this existentially quantified formula in zone $\infty$ by $\mathrm{CF}\left(\mathcal{B}_{1}, \mathcal{B}_{2}\right)$. Focusing on $\mathrm{CF}\left(\mathcal{B}_{1}, \mathcal{B}_{2}\right)$ will result on three premises:

$$
P_{1}: \vdash_{\Sigma} \Gamma,(\text { in }(\mathrm{e}, \mathrm{e}, \mathrm{id} 2))^{\perp} \quad P_{2}: \vdash_{\Sigma} \Gamma^{\prime},(\text { out }(\mathrm{e}, \mathrm{id} 1))^{\perp} \quad P_{3}: \vdash_{\Sigma} \Gamma^{\prime \prime}, \perp
$$

\footnotetext{
${ }^{6}$ This is always possible due to renaming and $\alpha$-equivalence.

${ }^{7}$ Note that, by the definition of substitution, the $r_{j}$ must be pairwise distinct. In contrast, $\mathrm{v}_{i}$ can be repeated in case a node contains more than one site.
} 
In order to prove $P_{1}$ and $P_{2}$ it must be the case that $\Gamma=\{$ in $(x, \mathrm{e}, \mathrm{id} 2)\}$ and $\Gamma^{\prime}=\{\operatorname{out}(y, \mathrm{id} 1)\}$. Since these are linear resources, they will not be in $\Gamma^{\prime \prime}$. The unification of the atoms in the initial rule will generate the substitutions $[\mathrm{e} / x]$ and $[\mathrm{e} / y]$, which will be propagated to the formulas in $\Gamma^{\prime \prime}$. While the substitution on $x$ will have no effect (each inner channel occurs only once in the encoded sequent, see Definition 5), the substitution on $y$ will rename an outer channel to a concrete edge which is connected to another component. It will instantiate variables in some control's list of links $L$, i.e., a control in $\mathcal{B}_{1}$ which was connected to an outer channel $y$ will become connected to a real edge e in $\mathcal{B}_{2}$.

The constants id1 and id2 guarantee that such channels belong to different bigraphs. Each time a focused derivation is performed on this formula, a connection will be made. All connections will have been made once the formula can no longer be derived.

It is also possible to perform the linking without using free variables and unification by first guessing a linking and then performing proof search to ensure that such a linking exists. Since the bigraphs are composable by assumption, a valid instantiation for the variables always exists. Our search procedure will have to check that all the in links of the outer bigraph have been instantiated in either view of the linking procedure.

The next step is to place $\mathcal{B}_{1}$ 's roots inside $\mathcal{B}_{2}$ 's sites. For this operation, we will need to reason on (and unify) subexponentials. The formula used for placement is constructed according to the sites available in $\mathcal{B}_{2}$. Let $L=\left\{l \mid l\right.$ :site(id2) $\left.\in \Gamma_{2}\right\}$; then: $\vdash_{\Sigma} \Gamma_{1}, \Gamma_{2},\left\{\left(?^{l} \operatorname{site}(i d 2)\right)^{\perp} \otimes\left(?^{l} \operatorname{root}(\text { id1 })\right)^{\perp} \otimes \perp \mid l \in L\right\}$ is derivable. We will henceforth denote this defined set of formulas as $\mathrm{PF}\left(\mathcal{B}_{1}, \mathcal{B}_{2}\right)$. A focused derivation on each of formulas in $\operatorname{PF}\left(\mathcal{B}_{1}, \mathcal{B}_{2}\right)$ will behave similarly to the case of $\mathrm{CF}$ : three premises will be generated.

$$
P_{1}: \vdash_{\Sigma} \Gamma, l:(\operatorname{site}(\mathrm{id} 2))^{\perp} \quad P_{2}: \vdash_{\Sigma} \Gamma^{\prime}, l:(\operatorname{root}(\text { id1 }))^{\perp} \quad P_{3}: \vdash_{\Sigma} \Gamma^{\prime \prime}, \perp
$$

And in order to prove $P_{1}$ and $P_{2}$ it is necessary that $\Gamma=\{l:$ site(id2) $\}$ and $\Gamma^{\prime}=\{r: \operatorname{root}(\mathrm{id} 1)\}$. Since $r$ is a variable, we obtain the substitution $[l / r]$ when proving $P_{2}$ which is propagated to $\Gamma^{\prime \prime}$. The variable $r$ represented a root in $\mathcal{B}_{1}$, while $l$ is the place where a site is located in $\mathcal{B}_{2}$. Note that $\Gamma^{\prime \prime}$ might have occurrences of $r$ as the label of other formulas, and by replacing it with $l$, we are in fact changing the location of some formulas. They are moved from the root $r$ in $\mathcal{B}_{1}$ to a location $l$ in $\mathcal{B}_{2}$, which is exactly what happens during composition of places. The subexponential signature also needs to be changed accordingly, and this is done by accumulating this substitution in $\Sigma$ 's $\sigma$.

Note that, since PF is a linear set, the connection of roots to nodes will only be complete once all formulas in the set are derived. Of course this will be composed of many focused phases.

We can now combine the operations of linking and placement to obtain a sequent whose derivation will result on a premise that encodes the composition of two bigraphs. We will require that such derivation is exhaustive, meaning that the formulas in $\infty$ are focused on until they can no longer be derived and there are no positive formulas in $\varepsilon$. 

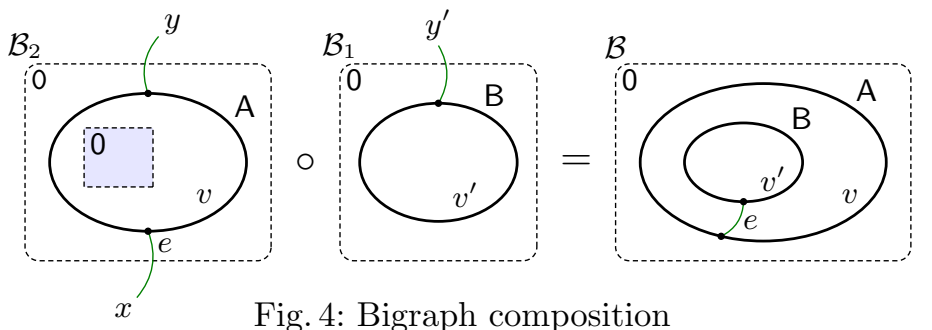

Fig. 4: Bigraph composition

Theorem 7 (Adequacy of bigraph composition). Let $\mathcal{B}_{1}$ and $\mathcal{B}_{2}$ be two bigraphs such that their composition $\mathcal{B}_{2} \circ \mathcal{B}_{1}=\mathcal{B}$ is well defined. Let $E\left(\mathcal{B}_{1}\right)=$ $\vdash_{\Sigma_{1}} \Gamma_{1}, E\left(\mathcal{B}_{2}\right)=\vdash_{\Sigma_{2}} \Gamma_{2}$ and $\Sigma=\Sigma_{2} \circ \Sigma_{1}$. Then the exhaustive focused derivation of the sequent $\vdash_{\Sigma} \Gamma_{1}, \Gamma_{2}, \mathrm{CF}\left(\mathcal{B}_{1}, \mathcal{B}_{1}\right), \operatorname{PF}\left(\mathcal{B}_{1}, \mathcal{B}_{2}\right)$ will have exactly one open premise $\vdash_{\Sigma} \Gamma, \infty: \Delta$ with $D\left(\vdash_{\Sigma} \Gamma\right)=\mathcal{B}$.

Proof (sketch). After exhaustive proof search, the failure to derive $\mathrm{CF}\left(\mathcal{B}_{1}, \mathcal{B}_{2}\right)$ and the derivation of all formulas in $\operatorname{PF}\left(\mathcal{B}_{1}, \mathcal{B}_{2}\right)$ means that there are no more in $(x, e$, id2), out $(y$, id1), site(id2) and root(id1) in the sequent. The fact that the bigraphs were compatible for composition in the first place, and the unique identifier in the atoms, guarantees that each of the predicates occurring in the sequent have the same multiplicity (number of inner links and sites of $\mathcal{B}_{2}$ must be the same as the number of outer links and roots of $\mathcal{B}_{1}$, respectively). The unifications during the derivations of CF's will ensure that outer links in $\mathcal{B}_{1}$ are renamed to the proper edges or outer links of $\mathcal{B}_{2}$. The unifications during the derivations of PF's will ensure that all elements inside of a root in $\mathcal{B}_{1}$ are correctly placed in the parent of the respective site in $\mathcal{B}_{2}$. Therefore, decoding the sequent without the $\infty$-formulas produces a bigraph with the desired structure.

As an example, consider the composition depicted in Figure 4. The encoding of each operand will be:

$$
\begin{aligned}
& E\left(\mathcal{B}_{1}\right): \quad \Gamma_{1}=\operatorname{out}\left(y^{\prime}, \text { id1 }\right), r_{0}^{\prime}: \operatorname{root}(\mathrm{id} 1), r_{0}^{\prime}: \operatorname{control}\left(\mathrm{B}, \mathrm{v}^{\prime},\left[y^{\prime}\right]\right) \\
& \Sigma_{1}=\left(\left\{r_{0}^{\prime}, \mathrm{v}^{\prime}, \infty\right\},\{\infty\},\left\{r_{0}^{\prime} \leq_{1} \mathrm{v}^{\prime}\right\}\right) \\
& E\left(\mathcal{B}_{2}\right): \quad \Gamma_{2}=\operatorname{in}(x, \mathrm{e}, \mathrm{id} 2), \text { out }(y, \mathrm{id} 2), r_{0}: \operatorname{root}(\mathrm{id} 2), \\
& \text { v:site(id2), } \left.r_{0} \text { :control(A, v, }[y, e]\right) \\
& \Sigma_{2}=\left(\left\{r_{0}, \mathrm{v}, 0, \infty\right\},\{\infty\},\left\{r_{0} \leq_{2} \mathrm{v} \leq_{2} 0\right\}\right)
\end{aligned}
$$

The formulas encoding the operation are:

$$
\begin{array}{ll}
\mathrm{CF}\left(\mathcal{B}_{1}, \mathcal{B}_{2}\right): & \infty: \exists e \cdot \operatorname{in}(e, e, \text { id } 2)^{\perp} \otimes \operatorname{out}(e, \text { id } 1)^{\perp} \otimes \perp \\
\mathrm{PF}\left(\mathcal{B}_{1}, \mathcal{B}_{2}\right): & \left\{\varepsilon:\left(?^{v} \operatorname{site}(\text { id } 2)\right)^{\perp} \otimes\left(?^{v} \operatorname{root}(\text { id } 1)\right)^{\perp} \otimes \perp\right\}
\end{array}
$$

The formula $\mathrm{CF}$ can only be derived once, indeed there is only one formula of each kind: in $(x, \mathrm{e}, \mathrm{id} 2)$ and out $\left(y^{\prime}, \mathrm{id} 1\right)$. As a result of this derivation, we will obtain the following substitution: $\left[\mathrm{e} / x, y^{\prime}\right]$. The derivation of the only formula in PF will provide the substitution $\sigma=\left[\mathrm{v} / r_{0}^{\prime}\right]$ for the subexponentials and formula labels. The resulting open sequent will be $\vdash_{\Sigma} \Gamma$, where:

$$
\Gamma=\varepsilon: \operatorname{out}(y, \mathrm{id} 2), r_{0}: \operatorname{root}(\mathrm{id} 2), r_{0}: \operatorname{control}(\mathrm{A}, \mathrm{v},[y, \mathrm{e}]), \mathrm{v}: \operatorname{control}(\mathrm{B}, \mathrm{v},[\mathrm{e}])
$$


$\Sigma=\left(\left\{r_{0}, \mathrm{v}, 0, \mathrm{v}^{\prime}, \infty\right\},\{\infty\},\left\{r_{0} \leq \mathrm{v} \leq \mathrm{v}^{\prime}\right\}\right)$.

\section{Conclusions, Related Work, and Perspectives}

Conclusions. Using the expressive power of subexponentials, we have given an adequate encoding of pure bigraphs in SEL. We were able to reason about the static structure of bigraphs with only the subexponential signature generated from the encoding. With the full sequent encoding of two bigraphs, we can easily simulate juxtaposition and we can emulate composition using a (focused) derivation in SELF.

Sorting. In [10, Chapter 6], Milner shows how to add sorts to links and places, and to define formation rules that will restrict the possible structures of a bigraph. Likewise the place and link graphs of a bigraph, the place and link sorts (and their formation rules) are orthogonal. In our encoding, we would use a multi-sorted version of SEL and assign the correct sorts for the variables and constants. The formation rules, by definition, are preserved under composition and juxtaposition, and as our encoding is adequate, they will also be preserved when operating on the meta-level.

Link sorting assigns sorts for each member of the arity of the controls and additionally to the inner and outer edges. The formation rules dictate what sorts of links a hyperedge may have in its extremities. In our encoding, we could transform each control $\mathrm{K}$ into a function symbol taking a list of arguments of appropriate type. In this case, the predicates control $\left({ }_{-}, \mathrm{K},\left[l_{1}, \ldots, l_{n}\right]\right)$ become control $\left(-, \mathrm{K}\left(l_{1}, \ldots, l_{n}\right)\right)$. Since $\mathrm{K}$ is always a constant in the encoding, this change is harmless. As for the inner and outer edges, we simply need to assign the correct types to the $x$ and $y$ variables. Unification will guarantee that the correct type is used on the connections when operating on the encoded bigraphs.

Place sorting assigns sorts for each control $K$ and formation rules determine what sorts can be contained in others. In our encoding, controls are assigned to vertices via the control predicate, but the containment relation is modeled by the relation between subexponentials. For this reason, adding sorts to places is a little more tricky then link sorting, but still possible. Sorts now have to be assigned to the $\mathrm{v}$ constants, representing nodes and, consistently, to the $\mathrm{v}$ subexponentials. Note that we bypass the sorts of controls and deal directly with the nodes (except sites and roots).

Bigraphs with Sharing. The pure bigraphs (with or without sorting) presented so far are sometimes not expressive enough; in particular, the tree-nature of the place graph is often too restrictive. In [16], the place graph was generalized from trees to arbitrary DAGs, allowing it to model an arbitrary Boolean combination of places. For our encoding, supporting DAGs is no harder than trees: our $\leq$ relation simply needs to be generalized to partial orders. This is easy: the meta-theory of SEL only requires $\leq$ to be a pre-order, so arbitrary directed graphs (acyclic or not) can be supported. The rest of the encoding remains unchanged.

Directed Bigraphs. A comparatively non-trivial extension is to move from an arbitrary (possibly sorted) hypergraph for the link graph to a directed link graph with link names as nodes [7]. While such a change of perspective does not need a 
modification of the encoding of bigraphs in this paper, adequacy is a bit more delicate. Specifically, the decoding function $D$ needs to know the exact signature of link names since the encoding represents link names as constants and not as linear resources. If we were to change the encoding to track the link names linearly, then the composition operation will need to be extended with new rules for pairing up the link names.

Perspectives. The most interesting followup to this work would be to develop the necessary reasoning on SEL encodings in order to get a bisimulation of bigraph reactions. In this way, we would have a full syntactical description of bigraphs with the advantage of having available automated proof theory tools.

Acknowledgment: This work was partially supported by the ERC Advanced Grant ProofCert.

\section{References}

1. M. Beauquier and C. Schürmann. A bigraph relational model. In $L F M T P$, volume 71 of EPTCS, pages 14-28, 2011.

2. L. Cardelli. Brane calculi. In $C M S B$, volume 3082 of $L N C S$, pages 257-278. Springer, 2004.

3. L. Cardelli and A. Gordon. Mobile ambients. In FOSSACS'98, volume 1378 of LNCS. Springer, 1998.

4. K. Chaudhuri. Classical and intuitionistic subexponential logics are equally expressive. In CSL, volume 6247 of LNCS, pages 185-199. Springer, Aug. 2010.

5. K. Chaudhuri. Undecidability of multiplicative subexponential logic. In 3rd LINEARITY, volume 176 of EPTCS, pages 1-8, July 2014.

6. V. Danos, J.-B. Joinet, and H. Schellinx. The structure of exponentials: Uncovering the dynamics of linear logic proofs. In Kurt Gödel Colloquium, volume 713 of LNCS, pages 159-171. Springer, 1993.

7. D. Grohmann and M. Miculan. Directed bigraphs. ENTCS, 173:121-137, 2007.

8. O. H. Jensen and R. Milner. Bigraphs and mobile processes (revised). Technical Report UCAM-CL-TR-580, University of Cambridge, Feb. 2004.

9. R. Milner. Pure bigraphs: Structure and dynamics. Information and Computation, 204(1):60 - 122, 2006.

10. R. Milner. The Space and Motion of Communicating Agents. Cambridge University Press, 2009.

11. V. Nigam. Exploiting non-canonicity in the sequent calculus. PhD thesis, Ecole Polytechnique, Sept. 2009.

12. V. Nigam and D. Miller. Algorithmic specifications in linear logic with subexponentials. In PPDP, pages 129-140, 2009.

13. V. Nigam, C. Olarte, and E. Pimentel. On subexponentials, focusing and modalities in concurrent systems. Draft Manuscript submitted for publication, 2015.

14. V. Nigam, E. Pimentel, and G. Reis. An extended framework for specifying and reasoning about proof systems. J. of Logic and Computation, 2014.

15. G. Păun. Membrane computing. In Handbook of Natural Computing, pages 13551377. Springer, 2012.

16. M. Sevegnani and M. Calder. Bigraphs with sharing. Theoretical Computer Science, 577:43-73, 2015. 